\title{
Simulation of the corrosion-induced damage on aluminum alloy 2024 specimens with equivalent surface notches
}

\author{
Nikolaos D. Alexopoulos, Nikoleta Siskou, Christina-Margarita Charalampidou \\ University of the Aegean, School of Engineering, Department of Financial and Management Engineering, 41 Kountouriots str, \\ 82132, Chios, Greece \\ nalexop@aegean.gr, bttp:/ / orcid.org/0000-0001-7851-1845
}

Stavros K. Kourkoulis

National Technical University of Athens, Department of Mechanics, Laboratory for Testing and Materials, Theocaris Building, 5 Heroes of Polytechnion Avenue, 157 73, Athens, Greece

\begin{abstract}
The effect of corrosion environment aggressiveness on the tensile mechanical properties degradation of AA2024-T3 was investigated. Tensile specimens were pre-corroded for various exposure times to different corrosive solutions, i.e., exfoliation corrosion (EXCO) and $3.5 \mathrm{wt} . \% \mathrm{NaCl}$. Then they were tested mechanically. In non-corroded specimens, surface notches of various depths were machined to simulate the degradation of the tensile mechanical properties due to the presence of artificial surface defects. A mechanical model was developed to correlate the corrosion-induced tensile ductility degradation due to pitting and possible hydrogen embrittlement with the equivalent artificially induced surface notches. The cases studied for this physical correlation were: a) EXCO exposure with artificial notches, b) EXCO with 3.5 wt. $\% \mathrm{NaCl}$ exposure and c) $3.5 \mathrm{wt} . \% \mathrm{NaCl}$ exposure with artificial notches. Higher correlation was noticed for short exposure times for all cases where the dominant degradation mechanism is slight pitting formation. It was found that $1 \mathrm{~h} \mathrm{EXCO}$ exposure is equivalent to $92 \mathrm{~h}$ exposure to $\mathrm{NaCl}$ solution regarding the tensile ductility degradation while $24 \mathrm{~h}$ EXCO exposure has the same effect on ductility decrease with a $240 \mu \mathrm{m}$ surface notch or $4000 \mathrm{~h}$ exposure to $\mathrm{NaCl}$ solution.
\end{abstract}

KEYworDs. Corrosion; Notches; Mechanical properties; Aluminum alloy.

\section{OPEN ACCESS}

Citation: Alexopoulos, N.D., Siskou, N., Charalampidou, C.M., Kourkoulis, S.K., Simulation of the corrosion-induced damage on aluminum alloy 2024 specimens with equivalent surface notches, Frattura ed Integrità Strutturale, 50 (2019) 342-353.

Received: 25.01.2019

Accepted: 27.05.2019

Published: 01.10.2019

Copyright: (c) 2019 This is an open access article under the terms of the CC-BY 4.0, which permits unrestricted use, distribution, and reproduction in any medium, provided the original author and source are credited.

\section{INTRODUCTION}

$\mathrm{D}$ uring the last decades attention has been paid by the aviation industries to the reduction of aircrafts maintenance costs. Damage tolerance evaluation for the skin and the fuselage of the aircraft is of major importance for the decrease of the inspection intervals and therefore of the respective maintenance costs. Among the greatest 
problems in maintenance and repair of aircraft structures is corrosion. The possibility that corrosion will interact with other forms of damage, e.g. fatigue cracks, impact etc. can result in significant loss of the structural integrity and may lead to fatal consequences, e.g. the Aloha Airlines accident. The synergetic interaction of corrosion and fatigue has a deteriorating effect on the mechanical performance of aeronautical aluminum alloys, mainly because of accelerated crack propagation [1]. The major damage mechanism on the corroded surface affecting the integrity of aircraft structures is the formation of pitting, e.g. [2-4]. Corrosion-induced pits initiate on the surface due to chemical or physical heterogeneities such as intermetallic particles, dislocations or mechanical damage and flaws, e.g. [5,6]. Wrought aluminum alloys used in aircraft applications, contain numerous intermetallic particles increasing substantially the mechanical properties (yield stress, fatigue crack growth etc.), nevertheless they play a pivotal role in the nucleation of pitting, e.g. [7,8].

Aluminum alloy 2024, is highly used in the aircraft industry due to its improved mechanical properties and high damage tolerance capability, nevertheless it shows high susceptibility to corrosion attack due to its microstructure [9-11]. Corrosion involves several electrochemical mechanisms; in acidified solutions, the basic anodic reaction is the metal dissolution while the cathodic reactions are oxygen and hydrogen reduction resulting from aluminum ion hydrolysis [2]. Dealloying of $S$ type $\left(\mathrm{Al}_{2} \mathrm{CuMg}\right)$ particles, that are the most common intermetallic phases in $2 \mathrm{xxx}$ aluminum alloys, leads to Cu-rich remnants within the clusters [12] that switch the anode reaction to the alloy matrix adjacent to the particle and eventually to the grain boundaries; thus, it assists the formation of sub-surface micro-cracks [13-15]. Cracking formation generally starts at local defects such as microstructural features inside the material, surface features such as notches or in-service damage process such as corrosion (e.g. pitting) that act as stress concentrators [16,17]. Accumulated corrosion damage can be noticed on aging aircrafts due to corrosion-induced embrittlement mechanisms. Hydrogen embrittlement phenomenon along with corrosion of aluminum alloys can lead to the rapid failure of the materials. Hydrogen is usually produced by surface corrosion reactions and afterwards diffuses into the material and is trapped at preferential sites [18] as shown in [19,20]. It can be adsorbed at crack tips or notches or diffuse ahead of cracks [21] that embrittles the material below the crack tip.

To face the corrosion-induced structural degradation issue, available data usually refer to accelerated laboratory tests. Many researchers focused on the development of damage functions to account for the corrosion assessment on the mechanical properties, e.g. [21-23]. The most common accelerated corrosion test used for the aluminum alloys of the $2 \mathrm{xxx}$ and $7 \mathrm{xxx}$ alloy series is the exfoliation corrosion (EXCO) test according to ASTM G34. It has been reported that 24 h exposure of the aluminum alloy 2024-T4 to the EXCO solution corresponds to nearly 6 years of natural exposure of the same structural element regarding its surface exfoliation [24]. Various mechanical tests had been carried out on AA2024-T3 to assess the effect of the corrosion damage on the material's structural integrity. Tensile and fatigue mechanical tests had been carried out in pre-corroded material, resulting to the mechanical properties degradation [25,26]. Corrosion of AA2024 was found to result in a moderate decrease of the strength properties (yield stress and ultimate tensile strength) with a significant reduction of tensile ductility [27,28]. According to Alexopoulos and Papanikos [29] the cross-sectional area of AA2024 specimens which was supposed to be unaffected by micro-cracks (referred as 'effective thickness'), decreases exponentially with increasing exposure time to EXCO solution due to the crack propagation mechanism that leads to higher corrosion penetration into the material. Corrosion exposure was found to have a negative effect on yield stress mainly due to the cross-sectional decrease at higher exposure times as well as on tensile ductility decrease due to the combination of hydrogen embrittlement in the low exposure times and decrease of the cross-section for the higher exposure times [30]. The reduction of the load carrying cross section of the specimens as well as the notch effects caused by pitting formation and exfoliated areas are sufficient to explain the moderate reduction of tensile strength properties.

The corrosion problem includes several degradation mechanisms and the damage could be described and analyzed as the sum of several parameters downgrading the mechanical properties. In order to better interpret the corrosion-induced damage, various mechanisms involved in the corrosion process should be taken into consideration; they may depend on material, temper, corrosive environment and exposure time. The aggressiveness of the corrosive environment is a significant parameter influencing corrosion damage evolution as well as the underlying corrosion mechanism. Recent investigations [31] indicate that in-service obtained corrosion damage correlates well to the one caused by a $3.5 \mathrm{wt} . \% \mathrm{NaCl}$ solution, with pitting density, depth and shape evolving with exposure time. Vasco et al. [32] performed correlations between corrosion damage from accelerated corrosion tests of varying aggressiveness by accounting for both, the metallographic features of corrosion damage and the mechanical properties of the corroded material. Correlations regarding geometrical metallographic features were found under dominance of pitting corrosion and up to 8 hours in EXCO solution; higher variations after the occurrence of pit coalescence and transition to dominance of exfoliation corrosion were presented.

The aim of the present work is to simulate the real corrosion-induced degradation of the mechanical properties due to corrosion surface pits and possible hydrogen embrittlement and to correlate it with the equivalent degradation by the artificially induced surface notches. Moreover, a comparison between the effects of the aggressiveness of the corrosion environment on the mechanical behavior of AA2024 specimens subjected to EXCO and $3.5 \mathrm{wt} \% \mathrm{NaCl}$ solution will be investigated. 


\section{EXPERIMENTAL PROCEDURE}

$\mathrm{T}$

he material used was a wrought aluminum alloy 2024-T3 which was received in sheet form of $3.2 \mathrm{~mm}$ nominal thickness. The weight percentage chemical composition of the alloy is $4.35 \% \mathrm{Cu}, 1.50 \% \mathrm{Mg}, 0.64 \% \mathrm{Mn}, 0.50 \%$ $\mathrm{Si}, 0.50 \% \mathrm{Fe}, 0.25 \% \mathrm{Zn}, 0.10 \% \mathrm{Cr}, 0.15 \% \mathrm{Ti}$ and $\mathrm{Al}$ rem. Tensile specimens were machined from the material sheet according to ASTM E8 specification with $12.5 \mathrm{~mm} \times 50 \mathrm{~mm}$ being the reduced cross section of the specimen. All the specimens were cut parallel to the longitudinal $(\mathrm{L})$ rolling direction of the material.

Reference specimens were tensile tested according to ASTM E8 specification, while two series of tensile specimens were exposed for various times to different laboratory corrosion environment, namely exfoliation corrosion (hereafter called EXCO solution) and $3.5 \mathrm{wt}$ \% sodium chloride (hereafter called $\mathrm{NaCl}$ solution) according to the specifications ASTM G34 and G44, respectively. The EXCO solution consisted of the following chemicals diluted in 11 distilled water; sodium chloride $(4.0 \mathrm{M} \mathrm{NaCl})$, potassium nitrate $\left(0.5 \mathrm{M} \mathrm{KNO}_{3}\right)$ and nitric acid $\left(0.1 \mathrm{M} \mathrm{HNO}_{3}\right)$. The results of the EXCO solution exposure have been performed and reported in a previous article of the authors [33]. The concentration of the $\mathrm{NaCl}$ solution consisted of $3.5 \mathrm{~g} \mathrm{NaCl}$ for each $96.5 \mathrm{ml}$ of water. The solution volume was calculated per exposure area of the specimens and it was constant for all specimens. In both experimental procedures, the specimens were cleaned with alcohol prior to corrosion exposure according to specification ASTM G1. Additionally, the specimens were masked with appropriate insulating PVC tape in order to be exposed only at the reduced surface area of approximate $55 \mathrm{~mm}$ in length. The experimental procedure was carried out in laboratory environmental conditions and at room temperature.

According to the literature [26], corrosion damage and hydrogen embrittlement is evident on the large surfaces of the tensile specimen's gauge length and not so intense on the side surfaces. Machining of the artificial notches was decided to be performed on the large surfaces of the tensile specimen, namely vertical to the loading axis. A drawing of the specimen with manufactured two surface notches can be seen in Fig.1. The two artificial notches facing one the other (on the same vertical level) can be well seen in the Figure as well as the maximum depth of $0.5 \mathrm{~mm}$ per notch. This notch depth per surface was the maximum depth of attack of corrosion products (pits and cracks) generated after approximating $72 \mathrm{~h}$ exposure time in exfoliation corrosion solution. In different specimens, notches with smaller depths were manufactured (ranging from 0.1 till $0.5 \mathrm{~mm}$ ) to incrementally simulate the corrosion surface damage on AA2024-T3 as well as the specimen's residual tensile strength and tensile ductility after the corrosion exposure.

Tensile tests were carried out in a servo-hydraulic Instron $8801100 \mathrm{kN}$ testing machine according to ASTM E8M specification, with a constant deformation rate of $3.3 \times 10^{-4} \mathrm{sec}$. An Instron extensometer $50 \mathrm{~mm} \pm 10 \mathrm{~mm}$ maximum travel was attached to the specimen's gauge length before the tensile test. A data logger was used during all tensile tests to store the values of load, displacement and axial strain in a computer. To get representative average values of the tensile properties, at least three tensile tests have been carried out per each test series. Evaluated properties were the conventional yield stress $R_{\mathrm{p} 0.2 \%}\left(0.2 \%\right.$ proof stress), ultimate tensile strength $R_{\mathrm{m}}$ and elongation at fracture $A_{\mathrm{f}}$.

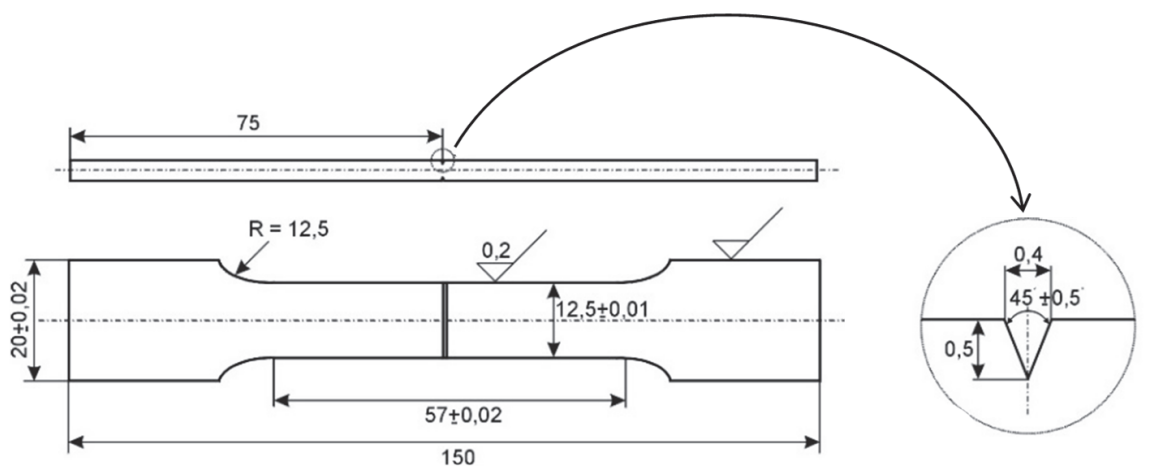

Figure 1: Sketch of the tensile specimen with machined two (upper and lower) surface notches on the large surfaces.

\section{RESULTS AND DISCUSSION}

\section{Surface characterization of pre-corroded specimens}

he exposure of AA2024 specimens to the corrosive environment (EXCO or 3.5 wt. $\% \mathrm{NaCl}$ solution in the present study) results in the deterioration of the surface of the specimens due to the nucleation of corrosioninduced surface pits, as can be seen in Fig.2. The depicted corroded area has dimensions of $12.5 \mathrm{~mm}$ x $55 \mathrm{~mm}$ being 
(a)

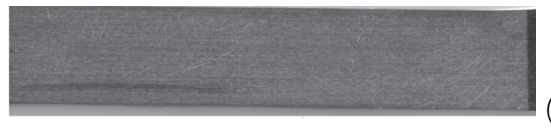

(d)

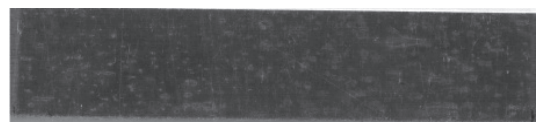

(b)

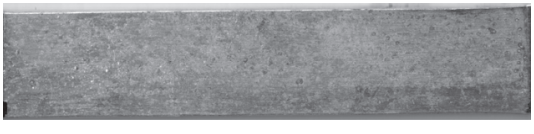

(e)

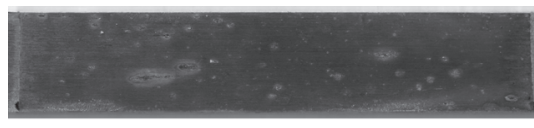

(c)

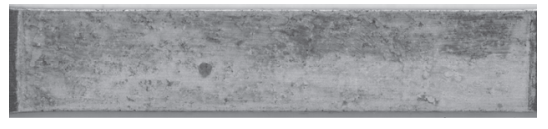

(f)

Figure 2: Typical photographs of AA2024-T3 pre-corroded tensile specimens exposed to the EXCO solution for (a)-(c) $2 \mathrm{~h}, 4 \mathrm{~h}$ and $24 \mathrm{~h}$ of EXCO solution and (d) - (f) $6 \mathrm{~h}, 168 \mathrm{~h}$ and $720 \mathrm{~h}$ of $3.5 \mathrm{wt} . \% \mathrm{NaCl}$ solution, respectively.

the width and the length of the exposed area of the specimens, respectively. For short exposure times to exfoliation corrosion solution and up to $2 \mathrm{~h}$, pitting formation on the corroded surfaces remains rather limited. With increasing exposure time to EXCO solution, an increase in the pitting density is evident. Corrosion damage initiates in the form of surface corrosion pits and evolves to the formation of micro-cracks and exfoliation areas due to the presence of intergranular corrosion. Regarding the corrosion environment of $3.5 \mathrm{wt} . \% \mathrm{NaCl}$ solution, it is evident that for the short exposure times the surface deterioration remains limited since pits were not identified after $6 \mathrm{~h}$ of exposure. However, the pitting density and size tend to increase with increasing exposure time; corrosion damage in the form of pits was observed after $168 \mathrm{~h}$ of exposure while more pits of higher diameter as well as pit coalescence are evident after $720 \mathrm{~h}$ exposure.

\section{Typical tensile curves of pre-corroded specimens}

Typical nominal tensile stress-strain curves for the investigated exposure times of AA2024-T3 to EXCO and 3.5 wt. \% NaCl solutions can be seen in Figs.3(a,b), respectively. The nominal stress calculation was based on the nominal cross-section of the tensile specimens, namely width $\mathrm{x}$ thickness $=12.5 \mathrm{~mm} \times 3.2 \mathrm{~mm}$. It can be noticed that for the short corrosion exposure times to EXCO solution and up to $2 \mathrm{~h}$, the values of axial nominal stress are not essentially influenced by the corrosion exposure while for higher exposure times, e.g. after $4 \mathrm{~h}$, a significant stress drop was noticed [33]. This stress drop was attributed to the decrease of the specimen's cross-section - due to the corrosion-induced micro-cracks formation - that withstand the applied mechanical loading, so called as "effective thickness" [29]. On the contrary, an essential decrease of tensile ductility is evident even for the very short exposure times, e.g. $0.5 \mathrm{~h}$ that can be attributed to the hydrogen embrittlement phenomenon. For even higher exposure times, both the axial nominal stress and axial nominal strain are essentially decreased. Regarding corrosion exposure to 3.5 wt. $\% \mathrm{NaCl}$ solution, no essential stress decrease was noticed even for the highest exposure time, e.g. 4200 h. However, elongation at fracture exhibited a significant degradation even for the very short exposure times such as $6 \mathrm{~h}$. It is worth mentioning that higher ductility degradation was observed at the time range between 6 and $168 \mathrm{~h}$, where pitting incubation takes place, as well as in the time range of 720 and $2184 \mathrm{~h}$, probably because of the change in the degradation mechanism, e.g. pit growth and coalescence.

Typical nominal tensile curves for the specimens of AA2024-T3 with machined surface notches can be seen in Fig.3c; the surface notch depth is a varying parameter. In the same figure, a reference tensile curve without any notches (black circles) was added for comparison. As can be seen, the surface notches act as stress concentrators and tend to decrease the tensile mechanical properties of the alloy and especially elongation at fracture that decreases in higher rates than the yield stress. An essential decrease of the axial nominal strain was noticed even for the low-depth notches; nevertheless, this was not the case for the strength properties that seem to be almost unaffected even for the notch depth of $0.30 \mathrm{~mm}$. For instance, the tensile curves of 0.10 and $0.15 \mathrm{~mm}$ notch depths showed quite the same behavior with the reference one with the exception of the significant loss in ductility. By increasing the notch depth, up to $0.50 \mathrm{~mm}$ in this work, a continuous elongation at fracture decrease was noticed (magenta circles) exhibiting extremely low tensile elongation at fracture values.

\section{Characterization of the fracture mechanism}

Stereoscopical examination on the fractured, pre-corroded specimens of AA2024-T3 (Fig.4) revealed that the pitting corrosion mechanism is extremely limited for the very short corrosion exposure times, i.e., up to $2 \mathrm{~h}$ to EXCO solution. It is evident that with increasing exposure time to EXCO solution, the pitting density tends to increase. A ductile fracture mechanism is evident from the $45^{\circ}$ slope of the fracture surface as can be seen in Figs.4(a,b). For the higher exposure times, e.g. $24 \mathrm{~h}$ in Fig.4c, it seems that the fracture paths follow a non-linear pattern and definitely from pit to pit. For the specimens exposed to $3.5 \mathrm{wt} . \% \mathrm{NaCl}$ solution, pitting was not as evident as observed in the respective EXCO specimens. Pitting density for relatively short exposure times of $6 \mathrm{~h}$ (Fig.4d) and $168 \mathrm{~h}$ (Fig.4e) was extremely small, while the pits substantially increased for higher exposure times, e.g. $720 \mathrm{~h}$ (Fig.4f). However, the $45^{\circ}$ slope of the fracture surface remains even after $720 \mathrm{~h}$ of exposure revealing a ductile fracture mechanism. 


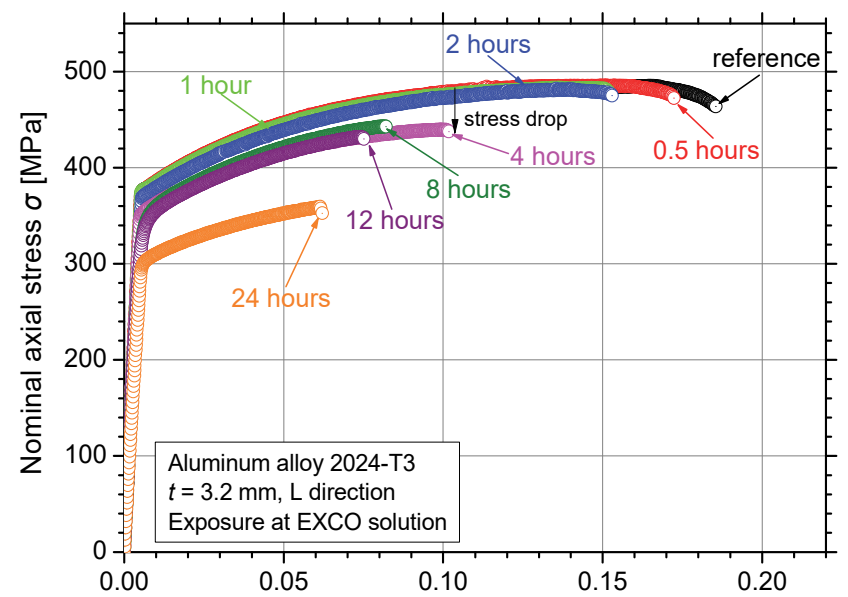

(a)

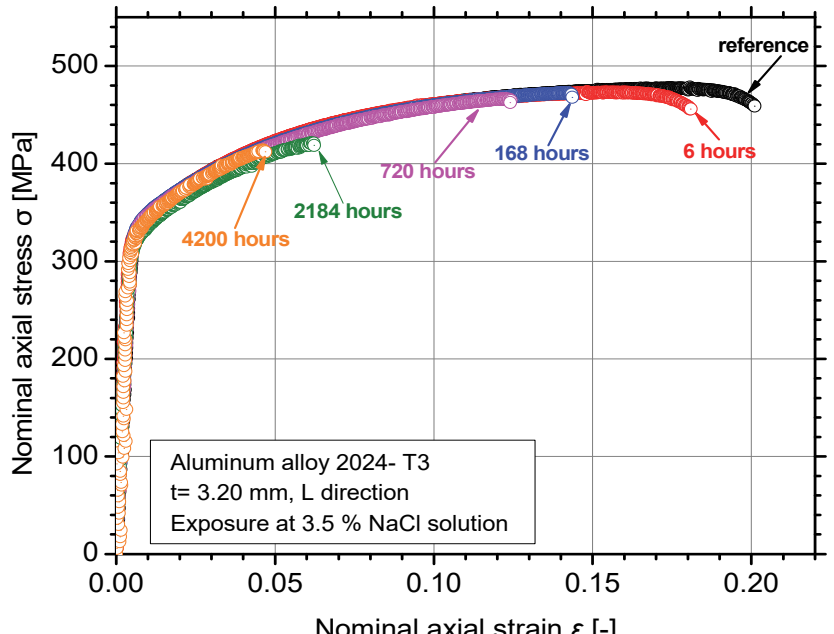

(b)

Nominal axial strain $\varepsilon[-]$

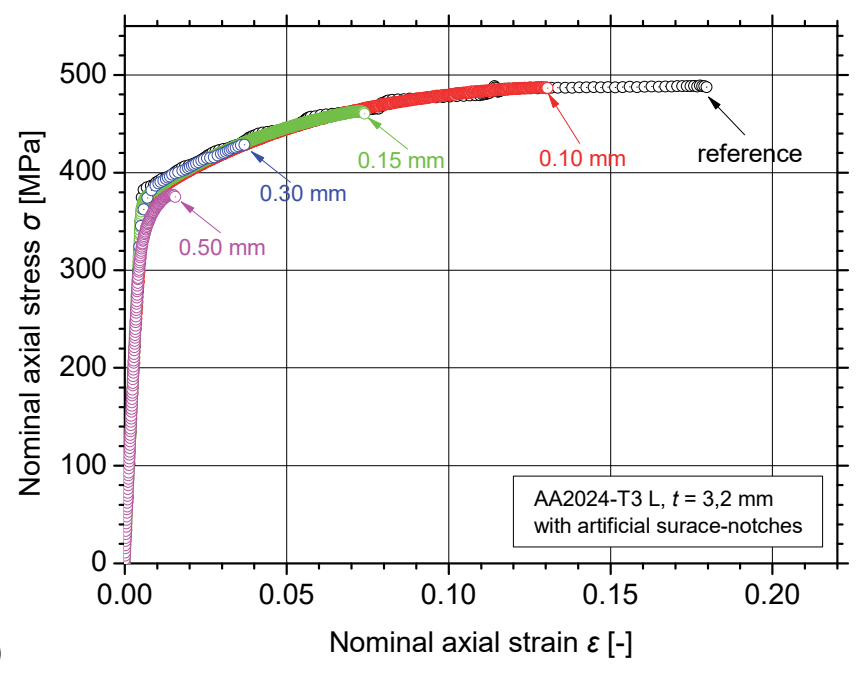

Figure 3: Typical tensile nominal stress - strain curves of AA2024-T3 after corrosion exposure for different exposure times to (a) EXCO solution, (b) 3.5 wt. $\% \mathrm{NaCl}$ solution and (c) varying depth of artificial surface notches.

(a)

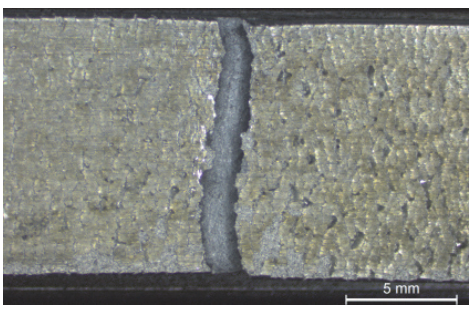

(d)

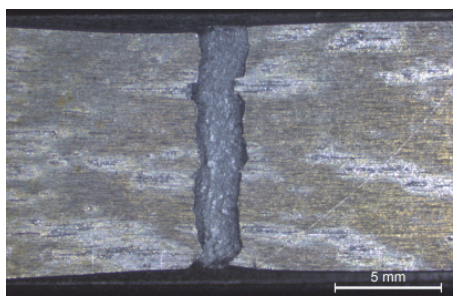

(b)

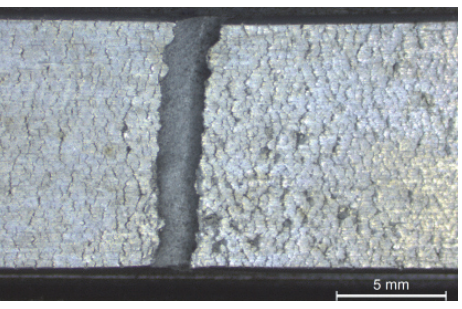

(c)
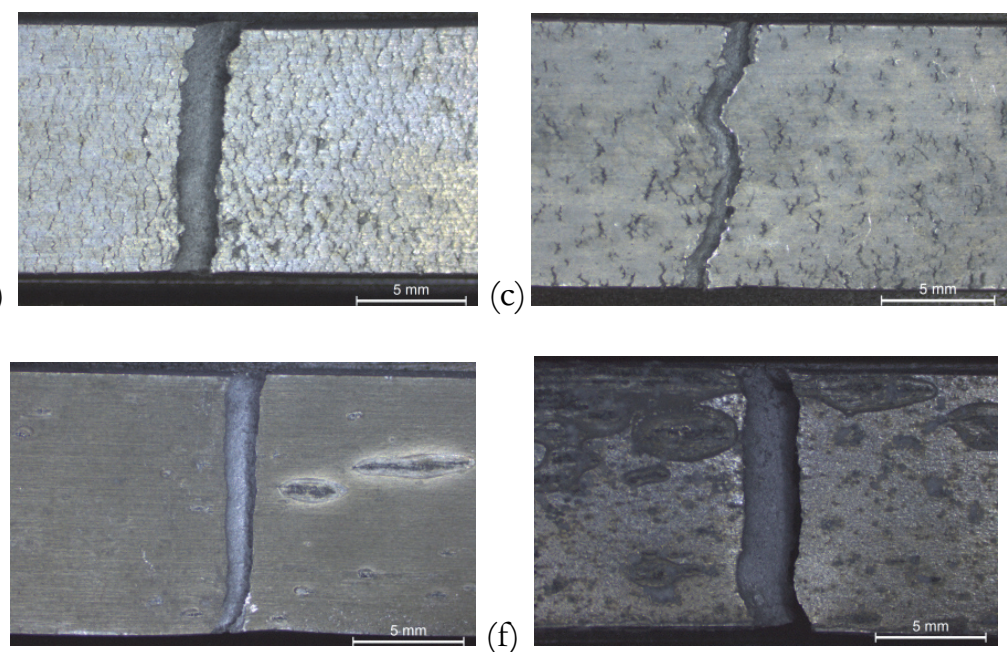

Figure 4: Typical photographs of AA2024-T3 pre-corroded tensile specimens exposed to the EXCO solution (a)-(c) for $2 \mathrm{~h}, 4 \mathrm{~h}$ and $24 \mathrm{~h}$, respectively and $3.5 \mathrm{wt} . \% \mathrm{NaCl}$ solution (d)-(f) for $6 \mathrm{~h}, 168 \mathrm{~h}$ and $720 \mathrm{~h}$, respectively. 
Effect on the conventional yield stress

The experimental results of the conventional yield stress $R_{\mathrm{p} 0.2 \%}$ (nominal values) for the (a) exposure times to EXCO solution, (b) exposure times to 3.5 wt. $\% \mathrm{NaCl}$ solution and (c) notch depths are presented in Fig.5. The results are presented in decreasing normalized values (y-axis) from the initial value of the conventional yield stress for comparison purposes. The black circles and dotted line represent the experimental results and fitting line of exposure to EXCO solution in both figures, the orange triangles and line corresponds to the fitting results of exposure to $3.5 \mathrm{wt}$. \% $\mathrm{NaCl}$ solution and the blue squares and dashed line to the respective results of notch depth. Regarding the comparison of conventional yield stress decrease between the two investigated corrosive environments, it is evident that the more aggressive solution (EXCO) leads to higher decrease of conventional yield stress up to $12 \mathrm{~h}$ of exposure. For the very short exposure times, the stress decrease seems to be almost the same for both investigated solutions; however specimens exposed to the mild corrosion solution $(3.5$ wt. $\% \mathrm{NaCl}$ ) needed more hours for the same degradation percentage, e.g. approximately $99 \%$ normalized decrease of $\mathrm{R}_{\mathrm{p} 0.2 \%}$ can be noticed after $168 \mathrm{~h}$ of exposure to $\mathrm{NaCl}$ solution as well as after $1 \mathrm{~h}$ of exposure to EXCO solution (Fig.5a). It is worth to mention that the 3.5 wt. $\% \mathrm{NaCl}$ solution does not reveal any exfoliation of the corrosion attacked material surfaces which could represent a more pronounced notch effect reducing the specimens' cross-sectional area. However, the decrease of conventional yield stress, remains limited for all the investigated corrosion exposure times of both investigated environments and does not exceed $40 \%$ at maximum and for the investigated exposure times. The

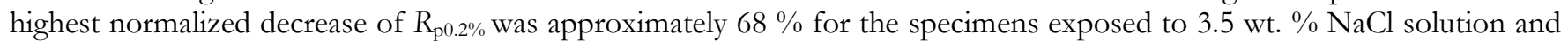
$79 \%$ for the specimens exposed to EXCO solution.

The comparison of the conventional yield stress decrease between the exposure to exfoliation corrosion solution and machined notch depths, is performed in Fig.5b. The specimens exposed to exfoliation corrosion exhibited higher conventional yield stress $R_{\mathrm{p} 0.2 \%}$ degradation than the specimens with the machined artificial surface notches up to $12 \mathrm{~h}$ of corrosion exposure as well as $0.3 \mathrm{~mm}$ notch depth. Corrosion exposure decreases the conventional yield stress with higher rates even from the short exposure times, e.g. $2 \mathrm{~h}$ where the pitting formation is limited, probably because of the

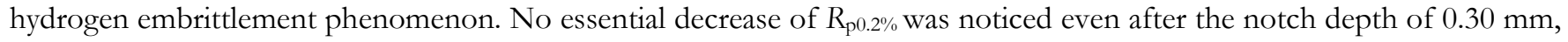
e.g. a $97 \%$ normalized decrease on $R_{\mathrm{p} 0.2 \%}$ was observed. For the short exposure times and low notch depth values, the contribution of EXCO and notch depth to the normalized property decrease is almost the same, e.g. the 0.1 and $0.15 \mathrm{~mm}$ notch depths resulted approximately in the same decrease as for the 0.5 and $1 \mathrm{~h}$ of exposure to EXCO solution; however, after $2 \mathrm{~h}$ corrosion exposure a higher decrease rate is noticed because of the synergetic effect of pitting corrosion and hydrogen embrittlement. Nevertheless, the machined notch depth of $0.50 \mathrm{~mm}$ yielded the same normalized conventional yield stress decrease as for the highest exposure time to EXCO, with the normalized decrease, reaching almost $80 \%$. The decrease in the conventional yield stress with increasing notch depth is well accepted since high notch depth values tend to increase the plasticity induced in front of the notch tip; plastic region increases with increasing stress level and general yielding at the reduced cross-section occurs for lower applied force level, thus resulting in lower nominal stress level.

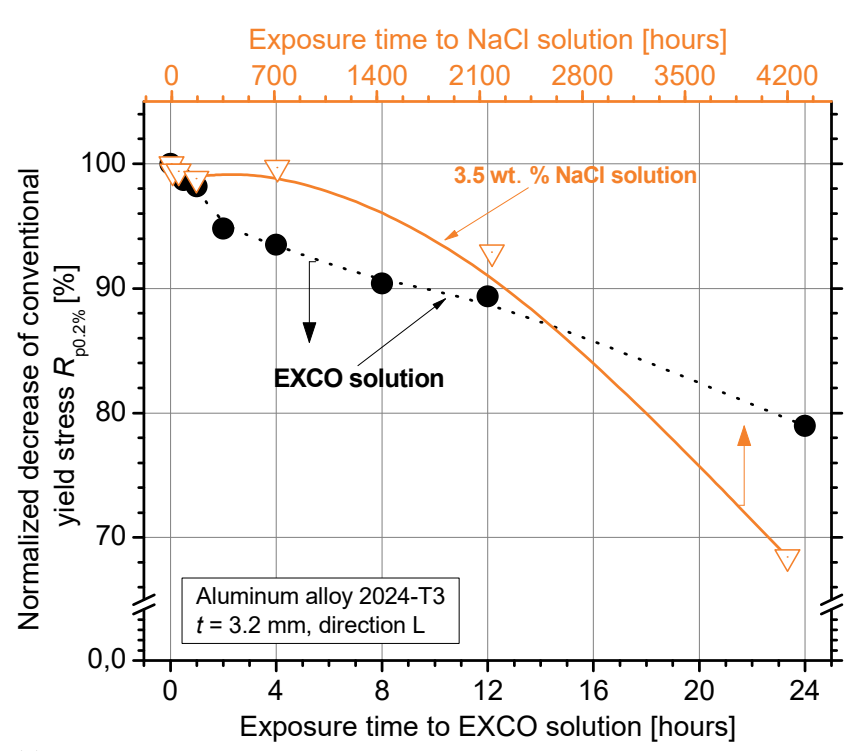

(a)

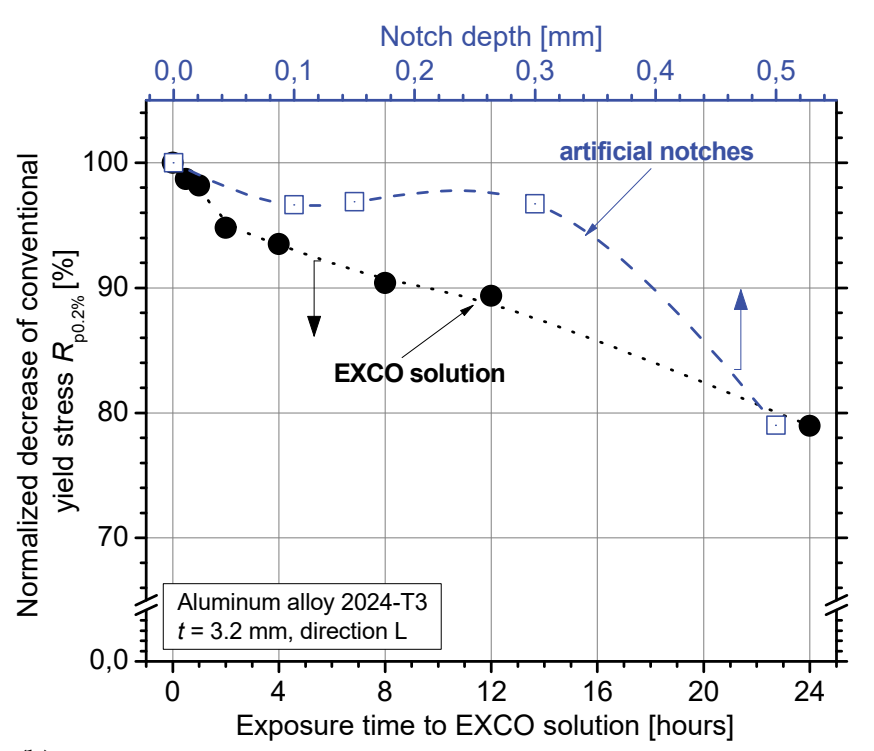

(b)

Figure 5: Normalized decrease of conventional yield stress $R_{\mathrm{p} 0.2 \%}$ values for the various exposure times of AA2024-T3 to the EXCO solution compared with (a) 3.5 wt. $\% \mathrm{NaCl}$ solution and (b) notch depths. 


\section{Effect on tensile ductility}

The results of the residual elongation at fracture $A_{\mathrm{f}}$ values can be seen in Fig.6 for the three investigated cases, namely the EXCO exposure, the $\mathrm{NaCl}$ exposure as well as the artificial notches. The results are presented in the form of normalized decrease from the initial value of $A_{\mathrm{f}}$ with double $\mathrm{X}$-axis for comparison reasons. Corrosion exposure has a deleterious effect on tensile ductility (elongation at fracture in the present case) even from the short exposure times to both investigated corrosive solutions. The normalized decrease of elongation at fracture $A_{\mathrm{f}}$ is almost the same for both solutions at short exposure times where the pitting formation remains limited; approximately $75 \%$ remaining percentage of the initial mechanical property was observed after only $1 \mathrm{~h}$ of exposure to EXCO solution while the same decrease was noticed for the case of exposure to 3.5 wt. \% NaCl solution after $48 \mathrm{~h}$ (Fig.6a). The same was observed for $2 \mathrm{~h}$ at EXCO and $168 \mathrm{~h}$ at 3.5 wt. $\% \mathrm{NaCl}$ with a normalized decrease value of $68 \%$. Nevertheless, higher exposure times are needed for the 3.5 wt. $\%$ $\mathrm{NaCl}$ solution in order to result in the same degradation percentage with the respective specimens exposed to EXCO solution. It should also be taken into consideration that the hydrogen embrittlement phenomenon takes place in this case, especially at the short exposure times in EXCO solution.

The respective results for the comparison between corrosion exposure to EXCO solution and artificial surface notches are presented in Fig.6b. It is evident that the normalized $A_{\mathrm{f}}$ decrease is not linear proportional to the notch depth nor to the exposure time increase in the reduced cross-section. An essential $A_{\mathrm{f}}$ decrease was observed even for the low-depth notches, e.g. approximately $70 \%$ normalized decrease for $0.10 \mathrm{~mm}$, as well as for the short exposure times such as $75 \%$ normalized decrease after $1 \mathrm{~h}$ of exposure. After $12 \mathrm{~h}$ of exposure to exfoliation corrosion solution, the $A_{\mathrm{f}}$ decrease seems to reach a plateau value and further corrosion exposure did not decrease the tensile ductility considerably while for the increasing notch depth it decreases continuously to extremely low values such as $6 \%$ for the $0.50 \mathrm{~mm}$ notch depth; this is evidence of maximum depth of attack of the surface corrosion-induced cracks [30]. It is well accepted that the ductility decrease from the short corrosion exposure times can be attributed to the hydrogen embrittlement phenomenon.
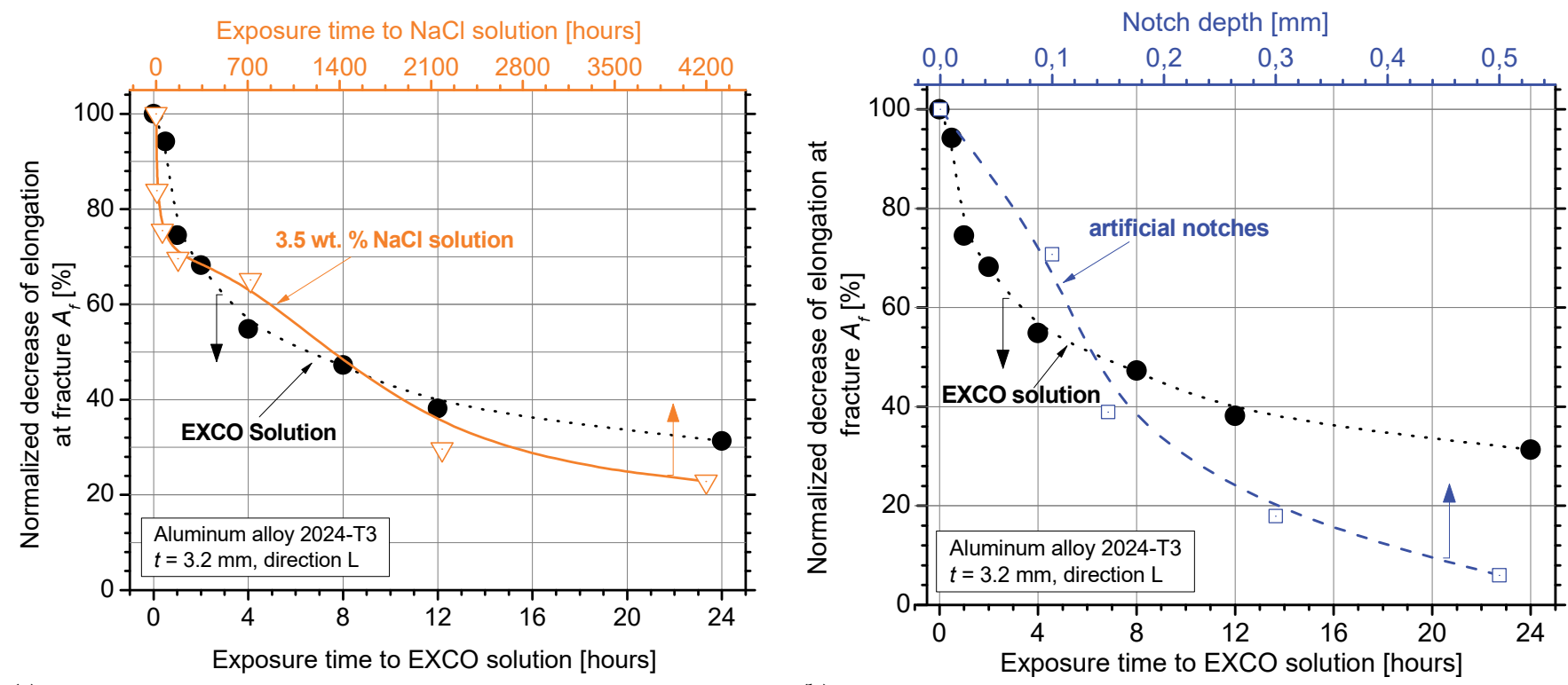

(a)

(b)

Figure 6: Normalized decrease of elongation at fracture $A_{f}$ values for the various exposure times to the EXCO solution of AA2024-T3 compared with (a) 3.5 wt. $\% \mathrm{NaCl}$ solution and (b) notch depth.

\section{Correlation of corrosion exposure times to notches regarding ductility degradation}

As shown in the previous two sections, the increase of the surface notch depth decreases the tensile elongation at fracture (ductility) of AA2024-T3 and to a lesser extent the conventional yield stress. From the experimental tensile test results of pre-corroded 2024-T3 specimens, an essential decrease in tensile ductility was noticed even for the short corrosion exposure times, for both investigated corrosive solutions as well as for small artificial notch depth values. Hence, it is obvious that the empirical correlation between the problem of corrosion-induced degradation and the equivalent problem with artificial surface notches should be assessed through the residual tensile ductility property.

The experimental results of the residual elongation at fracture $A_{\mathrm{f}}$ of the pre-corroded in EXCO solution tensile specimens of AA2024-T3 can be seen in Fig.7. In the same figure, the exponential decrease curve fitting was plotted as well. For the 
conversion of the total depth of the surface notches in equivalent exposure time to corrosion solution, an empirical coefficient was devised. The value of this coefficient was selected such as to 'tailor' the equivalent ductility decrease curve of the surface notches in order to take approximate values with the experimental ductility decrease curve of the pre-corroded specimens. The empirical correlation factor $m$ in $[\mathrm{h} / \mathrm{mm}]$ was calculated based on the following equation to correlate the available elongation at fracture test results as:

$$
\text { equivalent notch depth }(\mathrm{mm})=\frac{\text { exposure time }(h)}{m} \text {. }
$$

Fig. 7 shows the correlation of the elongation at fracture decrease induced by the exposure to EXCO solution as well as by the presence of the surface notches. The best calculation results were found by using the value $m=20$ for the empirical coefficient and the simulation of the ductility decrease curve of the investigated AA2024-T3 specimens for the short exposure times, where the synergy of pitting formation and hydrogen embrittlement is the dominant degradation mechanism. By using this coefficient value, it is obvious that the results of the artificial notch depths are very close to the experimental values for the short corrosion exposure times and up to $2 \mathrm{~h}$. It seems that the total notch depth of $0.10 \mathrm{~mm}$ corresponds to $2 \mathrm{~h}$ of exfoliation corrosion regarding the elongation at fracture decrease. On the other hand, the empirical coefficient that better simulates the tensile ductility decrease for the long exposure times, where the exfoliation of the corroded surfaces along with hydrogen embrittlement are the responsible mechanisms for the elongation at fracture decrease, was found to be approximate $m=100$. For instance, a total depth of $150 \mu \mathrm{m}$ surface notch results in the same $A_{\mathrm{f}}$ decrease as for $15 \mathrm{~h}$ of exposure to exfoliation corrosion solution. Summarizing the available test results, the factor $m$ takes values as:

$$
m_{\text {EXCO to notch }}=\left\{\begin{array}{rc}
20, & 0<t<4 \mathrm{~h}, \\
100,16<t<48 \mathrm{~h}, & \text { pitting and hydrogen emrittlement }
\end{array}\right.
$$

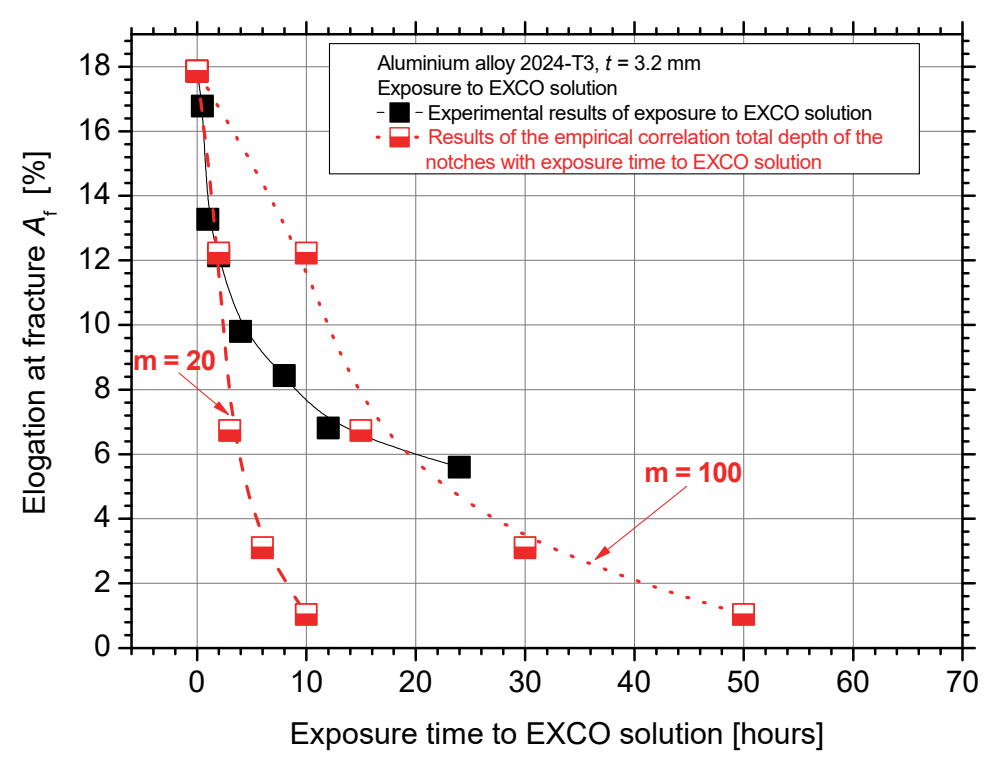

Figure 7: Correlation of the elongation at fracture $A_{\mathrm{f}}$ decrease due to exposure to the EXCO solution as well as to the presence of the artificial surface notches.

Fig. 8 shows the correlation of elongation at fracture decrease resulting from the exposure to 3.5 wt. \% $\mathrm{NaCl}$ solution, along with the respective results from the artificial surface notches. It can be noticed that the residual elongation at fracture of the two different cases is well correlated for the short exposure times by using a coefficient value $m=500$. This means that $50 \mathrm{~h}$ of exposure to $3.5 \mathrm{wt} . \% \mathrm{NaCl}$ solution results in the same elongation at fracture decrease as for $0.1 \mathrm{~mm}$ surface notch depth. The pitting corrosion mechanism is responsible for the ductility decrease for the short exposure times. However, a different value of this coefficient should be used, $m=15000$, in order to obtain good correlation for the long exposure times and high notch depths, wherein the effect of micro-cracks formation due to pit growth and coalescence is responsible for the $A_{\mathrm{f}}$ degradation. Hence, the following equation can be drawn by the findings of the results of the experimental protocols:

$$
m_{\mathrm{NaCl} \text { to notch }}=\left\{\begin{aligned}
500, \quad 0 & <t<400 \mathrm{~h}, & & \text { incubation of pits } \\
15.000,2000 & <t<8000 \mathrm{~h} & & \text { pit growth }
\end{aligned}\right.
$$




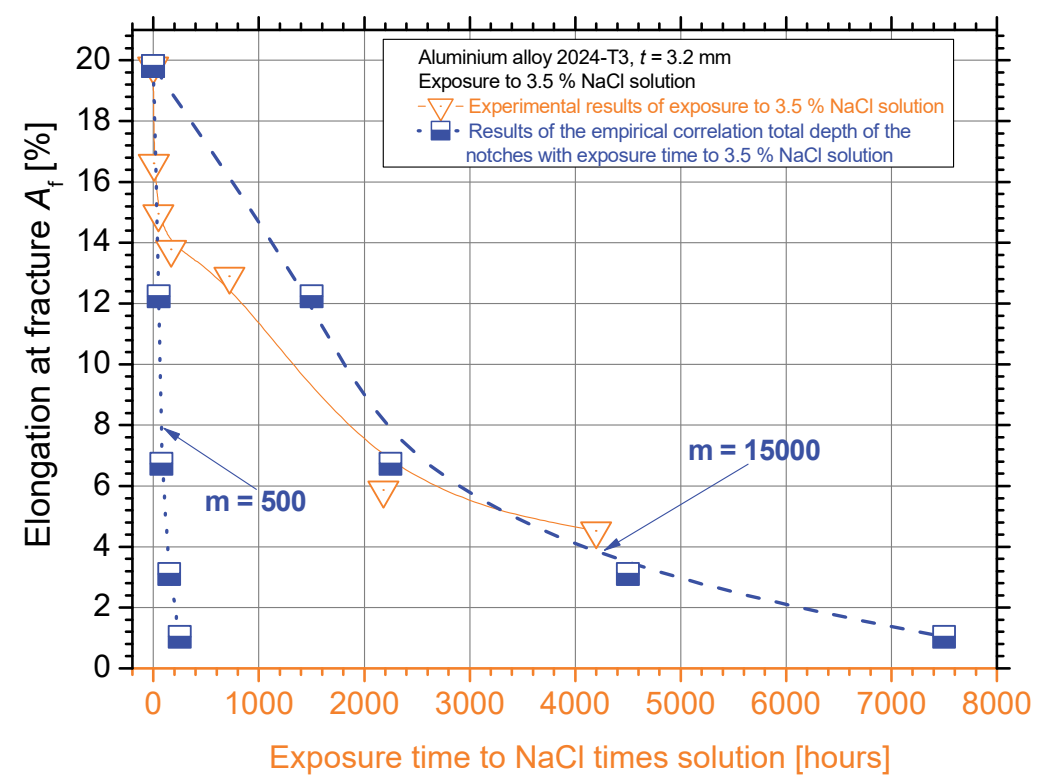

Figure 8: Correlation of the elongation at fracture $A_{\mathrm{f}}$ decrease due to exposure to the $3.5 \mathrm{wt} . \% \mathrm{NaCl}$ solution as well as to the presence of the artificial surface notches.

Fig.9 shows the correlation results of the effect of the two corrosive environments, i.e. EXCO and 3.5 wt. \% $\mathrm{NaCl}$ solutions, with regard to the same corrosion-induced tensile ductility decrease. To this end, for the conversion of exposure times to EXCO solution to the respective times of exposure to $\mathrm{NaCl}$ solution, the $n$ coefficient in [-] is formulated as:

$$
n_{\mathrm{EXCO} \text { to } \mathrm{NaCl}}=\frac{\text { exposure time to } \mathrm{NaCl}(\mathrm{h})}{\text { exposure time to } \operatorname{EXCO}(\mathrm{h})}
$$

The best calculation results were found by using the value $n=92$ for the empirical coefficient and for the simulation of the tensile ductility decrease curve of the investigated specimens for short exposure times regime. The same rate decrease is evident between the two different corrosive solutions at the short exposure times where slight pitting formation is the dominant degradation mechanism. By using this coefficient value, it is obvious that the results of the exfoliation corrosion are very close to the experimental values of exposure to $3.5 \mathrm{wt} \% \mathrm{NaCl}$ up to $1 \mathrm{~h}$ of exposure and $14 \%$ elongation at fracture. Thus, it can be concluded that $1 \mathrm{~h}$ of exposure to EXCO solution is equivalent to $92 \mathrm{~h}$ of exposure to $3.5 \mathrm{wt}$. \% $\mathrm{NaCl}$ solution regarding the $A_{\mathrm{f}}$ degradation. For higher exposure times, there is no correlation between the corrosive environments regarding the ductility decrease since the corrosion-induced degradation mechanism is different.

By exploiting this empirical tool, the design engineer could estimate the equivalent surface notches problem (fictitious notch) with the true problem of corrosion exposure of AA2024-T3. This might be a very useful tool as through experimental data or through finite element calculations, the design engineer could estimate the residual mechanical properties of the sheet alloy for maintenance and repair actions.

It is obvious that the proposed empirical correlation for 2024-T3 gives reliable results for short exposure duration in exfoliation corrosion solution. In short corrosion exposure times, the degradation mechanism of ductility has been correlated in the open literature with the hydrogen embrittlement. To this end, the above-mentioned empirical correlation is suggested to be exploited for exposure times higher than $2 \mathrm{~h}$, where the first surface pits and sub-sequent micro-cracks are beginning to be formed in the investigated cross-section of the AA2024-T3.

\section{CONCLUSIONS}

$\mathrm{S}$ ummarizing the findings of the present experimental study, it could be concluded that surface pitting corrosion remains limited for the short exposure times to both corrosive environments, e.g. up to $2 \mathrm{~h}$ in EXCO and $168 \mathrm{~h}$ in $\mathrm{NaCl}$ solution; a substantial increase in pitting density and size was observed with increasing exposure times for both investigated solutions. Moreover, all investigated tensile mechanical properties of AA2024-T3 are exponentially decreasing with increasing exposure time to corrosive solutions as well as surface notch depth; tensile ductility decreases with higher 


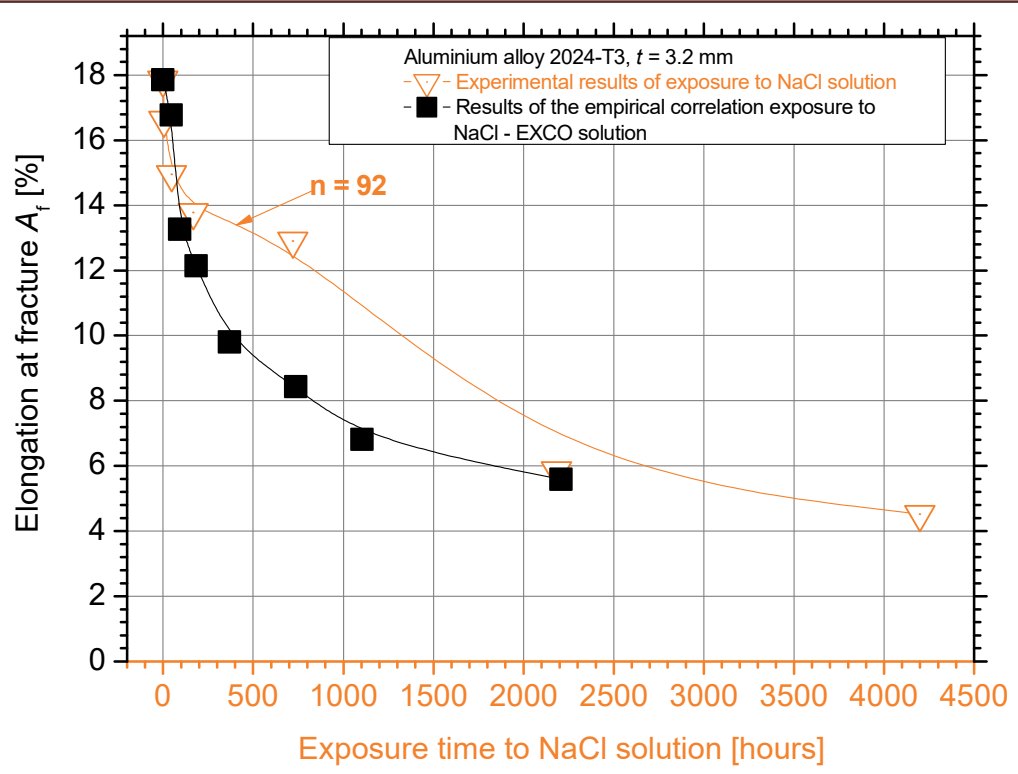

Figure 9: Correlation of the elongation at fracture $A_{f}$ decrease due to exposure to the EXCO solution as well as to 3.5 wt. $\% \mathrm{NaCl}$ solution.

rates than conventional yield stress for both, EXCO and 3.5 wt. $\% \mathrm{NaCl}$ solutions as well as for artificial surface notches. In addition, it was shown that a ductile fracture mechanism is evident from the $45^{\circ}$ slope of the fracture surface for up to $4 \mathrm{~h}$ for the specimens exposed to EXCO solution and even after $720 \mathrm{~h}$ for the specimens exposed to $\mathrm{NaCl}$ solution; however, for higher exposure times to EXCO solution, the fracture path seems to follow the surface pits.

Another interesting finding of the study is that the more aggressive environment (EXCO) results in higher decrease of the conventional yield stress, especially for the short exposure times. Higher exposure times to 3.5 wt. $\% \mathrm{NaCl}$ solution are needed for the same degradation of $R_{\mathrm{p} 0.2 \%}$ as for the EXCO solution, e.g. approximately $99 \%$ normalized $R_{\mathrm{p} 0.2 \%}$ decrease was noticed after $1 \mathrm{~h}$ of exposure to EXCO solution while the same decrease is evident after $168 \mathrm{~h}$ of exposure to $\mathrm{NaCl}$ solution. The specimens exposed to exfoliation corrosion exhibited higher conventional yield stress $R_{\mathrm{p} 0.2 \%}$ degradation than the specimens with the machined artificial surface notches, especially for the short exposure times and low depth of notches. However, the highest notch depth of $0.50 \mathrm{~mm}$ resulted in the same normalized decrease, approximately $80 \%$, as for the highest exposure time.

An empirical coefficient was introduced for the correlation between the corrosion-induced tensile ductility degradation with the equivalent artificially induced surface notches. Three cases were investigated: (a) EXCO exposure with artificial notches, (b) EXCO exposure with 3.5 wt. $\% \mathrm{NaCl}$ exposure and (c) 3.5 wt. $\% \mathrm{NaCl}$ exposure with artificial notches. Higher correlation regarding the ductility decrease was noticed for the short exposure times, where the slight pitting formation as well as hydrogen embrittlement for the case of EXCO solution are the dominant degradation mechanisms, and low-depth notches for all investigated cases. The best correlation between exposure to EXCO solution and artificial surface notches was found by using $m=20$ for the short exposure times where the main degradation mechanisms are the pitting formation along with hydrogen embrittlement; thus, a total notch depth of $0.10 \mathrm{~mm}$ corresponds to $2 \mathrm{~h}$ of exfoliation corrosion with regard to the same tensile ductility degradation. For the case of correlation between 3.5 wt. \% $\mathrm{NaCl}$ solution and artificial notches, the coefficient value that better simulates the corrosion-induced ductility decrease was found to be $m=500$ for exposure times less than $400 \mathrm{~h}$, where the incubation of pits is the dominant degradation mechanism; hence, $100 \mu \mathrm{m}$ surface notch depth results in the same elongation at fracture decrease as for $50 \mathrm{~h}$ of exposure to 3.5 wt. $\% \mathrm{NaCl}$ solution. For the case of correlation between exposure to EXCO and $3.5 \mathrm{wt} . \% \mathrm{NaCl}$ solutions, it is proposed that $1 \mathrm{~h} \mathrm{EXCO}$ exposure is equivalent to $92 \mathrm{~h}$ exposure to $\mathrm{NaCl}$ solution regarding tensile elongation at fracture decrease.

\section{REFERENCES}

[1] Menan, F., Henaff, G. (2009). Influence of frequency and exposure to a saline solution on the corrosion fatigue crack growth behavior of the aluminum alloy 2024, Int. J. Fatigue, 31, pp. 1684-1695.

DOI: 10.1016/j.ijfatigue.2009. 02.033 
[2] Davis, J.R. (1999). Corrosion of Aluminum and Aluminum Alloys, ASM International Materials Park, United States.

[3] Chen, G.S., Gao, M., Wie, R.P. (1996). Microconstituent-induced pitting corrosion in aluminum alloy $2024-T 3$. Corrosion, 52, pp. 8-15. DOI: 10.5006/1.3292099.

[4] Szklarska-Smialowska, Z. (1999). Pitting corrosion of aluminum. Corros. Sci., 41, pp. 1743-1767. DOI: 10.1016/ S0010-938X (99)00012.-8.

[5] Shreir, L.L., Jarman, R.A., Burstein, C.T. (1994). Corrosion: metal/environmental reactions, Oxford: Butterworth \& Heinemann Ltd. DOI: 10.1016/C2013-0-04015-7.

[6] Strehblow, H.H. (1995). Mechanisms of pitting corrosion, In: Corrosion mechanisms in theory and practice, New York, Marcel Dekker Inc.; pp. 201-238.

[7] Birbilis, N., Cavanaugh, M.K., Buchheit, R.G. (2006). Electrochemical behavior and localized corrosion associated with $\mathrm{Al}_{7} \mathrm{Cu}_{2} \mathrm{Fe}$ particles in aluminum alloy 7075-T651. Corros. Sci., 48, pp. 4202-4215.

DOI: $10.1016 /$ j.corsci.2006. 02.007.

[8] Boag, A., Taylor, R.J., Muster, T.H., Goodman, N., McCulloch, D., Ryan, C., Rout, B., Jamieson, D., Hughes, A.E. (2010). Stable pit formation on AA2024-T3 in a NaCl environment. Corros. Sci., 52, pp. 90-103.

DOI: $10.1016 /$ j.corsci.2009.08.043.

[9] Boag, A., Hughes, A.E., Glenn, A.M., Muster, T.H., McCulloch, D. (2011). Corrosion of AA2024-T3 part I: localised corrosion of isolated IM particles. Corros. Sci., 53, pp.17-26. DOI: 10.1016/j.corsci.2010.09.009.

[10] Blanc, C., Lavelle, B., Mankowski, G. (1997). The role of precipitates enriched with copper on the susceptibility to pitting corrosion of the 2024 aluminum alloy. Corros. Sci., 39, pp. 495-510. DOI: 10.1016/S0010-938X (97) 86099-4.

[11] DeRose, J.A., Suter, J., Bałkowiec, T., Michalski, A., Kurzydlowski, K.J., Schmutz, P. (2012). Localised corrosion initiation and microstructural characterization of an $\mathrm{Al} 2024$ alloy with a higher $\mathrm{Cu}$ to $\mathrm{Mg}$ ratio. Corros. Sci., 55, pp. 313-325. DOI: 10.1016/j.corsci.2011.10.035.

[12] Shi, H., Tian, Z., Hu, T. Liu, F. Han, E.H., Taryba, M. Lamaka, S.V. (2014). Simulating corrosion of $\mathrm{Al}_{2} \mathrm{CuMg}$ phase by measuring ionic currents, chloride concentration and pH. Corros. Sci., 88, pp. 178-186.

DOI: 10.1016/j.corsci. 2014.07.021.

[13] Hughes, A.E., Boag, A., Glenn, A.M., McCulloch, D., Muster, T.H., Ryan, C., Luo, C., Zhou, X., Thompson, G.E. (2011). Corrosion of AA2024-T3 part II: co-operative corrosion. Corros. Sci., 53, pp. 27-39. DOI: $10.1016 /$ j.corsci.2010.09.030.

[14] Buchheit, R.G., Grant, R.P., Hlava, P.F., Mckenzie, B., Zender, G.L. (1997). Local dissolution phenomena associated with $\mathrm{S}$ phase $\left(\mathrm{Al}_{2} \mathrm{CuMg}\right)$ particles in aluminum alloy2024-T3, J. Electrochem. Soc., 144, pp. 2621-2628. .DOI: $10.1149 / 1.1837874$.

[15] Hashimoto, T., Zhang, X., Zhou, X., Skeldon, P., Haigh, S.J., Thompson, G.E. (2016). Investigation of dealloying of $\mathrm{S}$ phase $(\mathrm{Al} 2 \mathrm{CuMg})$ in $\mathrm{AA} 2024-\mathrm{T} 3$ aluminium alloy using high resolution 2D and 3D electron imaging. Corros. Sci., 103, pp. 157-164. DOI: 10.1016/j.corsci.2015.11.013.

[16] Lyon, K.N., Marrow, T.J., Lyon, S.B. (2015). Influence of milling on the development of stress corrosion cracks in austenitic stainless steel. J. Mater. Process. Technol., 218, pp. 32-37. DOI: 10.1016/j.jmatprotec.2014.11.038.

[17] Ishihara, S., Saka, S., Nan, Z.Y., Goshima, T., Sunada, S. (2005). Prediction of corrosion fatigue lives of aluminium alloy on the basis of corrosion pit growth law. Fatigue Fract. Eng. Mater. Struct., 29, pp. 472-480.

DOI: $10.1111 /$ j.1460-2695.2006.01018.x.

[18] Azofeifa, D.E., Clark, N., Amador, A., Saenz, A. (1997). Determination of hydrogen absorption in Pd coated Al thin films. Thin Solid Films, 300, pp. 295-298. DOI: 10.1016/S0040-6090(96)09493-X.

[19] Kamoutsi, H. (2004), "Corrosion-induced hydrogen embrittlement in high-strength aluminum alloys", PhD thesis, Department of Mechanical and Industrial Engineering, University of Thessaly, Volos.

[20] Kamoutsi, H., Haidemenopoulos, G., Bontozoglou, V., Pantelakis, S.G. (2006). Corrosion-induced hydrogen embrittlement in aluminum alloy 2024, Corros. Sci., 48, pp. 1209-1224. DOI: 10.1016/j.corsci.2005.05.015

[21] Lynch, S.P. (2013). Mechanisms and kinetics of environmentally assisted cracking: current status, issues, and suggestions for further work, Metall. Mater. Trans. A, 44, pp. 1209-1229. DOI: 10.1007/s11661-012-1359-2.

[22] Liao, M., Bellinger, N.C., Komorowski, J.P. (2003). Modeling the effects of prior exfoliation corrosion on fatigue life of aircraft wing skins. Int. J. Fatigue, 25, pp. 1059-1067. DOI: 10.1016/j.ijfatigue.2003.08.005.

[23] Chen, G.S., Wan, K.C., Gao, M., Wei, R.P., Flournoy, T.H. (1996). Transition from pitting to fatigue crack growthmodeling of corrosion fatigue crack nucleation in a 2024-T3 aluminum alloy. Mater Sci. Eng. A, 219 , pp. 126-132. DOI: 10.1016/S0921-5093(96)10414-7.

[24] Sprowls, D.O., Walsh, J.D., Shumaker, M.B. (1972). Simplified exfoliation testing of aluminum alloys, ASTM STP 516. DOI: $10.1520 /$ STP35415S. 
[25] Jones, K., Hoeppner, D.W. (2006). Prior corrosion and fatigue of 2024-T3 aluminum alloy. Corros. Sci., 48, pp. $3109-$ 3122. DOI: $10.1016 /$ j.corsci.2005.11.008.

[26] Kermanidis, A.T., Petroyiannis, P.V., Pantelakis, S.G. (2005). Fatigue and damage tolerance behaviour of corroded 2024 T351 aircraft aluminum alloy. Theor. Appl. Fract. Mech., 43, pp. 121-132. DOI: 10.1016/j.tafmec.2004.12.008.

[27] Pantelakis, S.G., Daglaras, P.G., Apostolopoulos, C.A., (2000). Tensile and energy density properties of 2024, 6013, 8090 and 2091 aircraft aluminum alloy after corrosion exposure. Theor. Appl. Fract. Mech., 33, pp. 117-134. DOI: $10.1016 /$ S0167-8442 (00)00007-0.

[28] Petroyiannis, P.V., Kermanidis, A.T., Kamoutsi, H., Pantelakis, S.G., Bontozoglou, V., Haidemenopoulos, G.N. (2005). Evidence on the corrosion induced hydrogen embrittlement of the 2024 aluminum alloy. Fatigue Fract. Eng. Mater. Struct., 28, p.p. 565-574. DOI: 10.1111/j.1460-2695.2005.00900.x.

[29] Alexopoulos, N.D., Papanikos, P. (2008). Experimental and theoretical studies of corrosion-induced mechanical properties degradation of aircraft 2024 aluminum alloy. Mater. Sci. Eng. A, 498, pp. 248-257. DOI: $10.1016 /$ j.msea. 2008.08.024.

[30] Alexopoulos, N.D., Dalakouras, C.J., Skarvelis, P., Kourkoulis, S.K. (2012). Accelerated corrosion exposure in ultrathin sheets of 2024 aircraft aluminium alloy for GLARE applications. Corros. Sci., 55, pp. 289-300. DOI: 10. 1016/j.corsci.2011.10.032.

[31] Gurin, M., Andrieu, E., Odemer, G., Alexis, J., Blanc, C. (2014). Effect of varying conditions of exposure to an aggressive medium on the corrosion behavior of the $2050 \mathrm{AlCuLi}$ alloy. Corros Sci., 85, pp. 455-470. DOI: 10.1016 /j.corsci.2014.04.042.

[32] Vasco, M.C., Chamos, A.N., Pantelakis, S.G. (2017). Effect of environment's aggressiveness on the corrosion damage evolution and mechanical behavior of AA 2024-T3. Fatigue Fract. Eng. Mater. Struct., 40, pp 1551-1561. DOI: $10.1111 /$ ffe.12651.

[33] Alexopoulos, N.D., Velonaki, Z., Stergiou, C.I., Kourkoulis, S.K. (2016). The effect of artificial ageing heat treatments on the corrosion-induced hydrogen embrittlement of 2024 (Al-Cu) aluminium alloy, Corros. Sci., 102, pp. 413-424. DOI: 10.1016/j.corsci.2015.10.034. 